\title{
TextImager as a Generic Interface to $\mathbf{R}$
}

\author{
Tolga Uslu ${ }^{1}$, Wahed Hemati ${ }^{1}$, Alexander Mehler ${ }^{1}$, and Daniel Baumartz ${ }^{2}$ \\ ${ }^{1,2}$ Goethe University of Frankfurt \\ ${ }^{1,2}$ TextTechnology Lab \\ ${ }^{1}$ uslu, hemati, mehler\}@em.uni-frankfurt.de \\ ${ }^{2}$ baumartz@stud.uni-frankfurt.de
}

\begin{abstract}
$R$ is a very powerful framework for statistical modeling. Thus, it is of high importance to integrate $\mathrm{R}$ with state-of-theart tools in NLP. In this paper, we present the functionality and architecture of such an integration by means of TextImager. We use the OpenCPU API to integrate $R$ based on our own $R$-Server. This allows for communicating with $R$-packages and combining them with TextImager's NLPcomponents.
\end{abstract}

\section{Introduction}

We introduced TextImager in (Hemati et al., 2016) where we focused on its architecture and the functions of its backend. In this paper, we present the functionality and architecture of $R$ interfaced by means of TextImager. For this purpose, we created a separate panel in TextImager for $R$-applications. In this panel, we combine state-of-the-art NLP tools embedded into TextImager with the the powerful statistics of $R$ (R Development Core Team, 2008). We use the OpenCPU API (Ooms, 2014) to integrate $R$ into TextImager by means of our own $R$-server. This allows for easily communicating with the built-in $R$-packages and combining the advantages of both worlds. In the case of topic detection (LDA), for example, the complete text is used as an input string to $R$. Thanks to TextImager's preprocessor, more information is provided about syntactic words, parts of speech, lemmas, grammatical categories etc., which can improve topic detection. Further, the output of $\mathrm{R}$ routines is displayed by means of TextImager's visualizations, all of which are linked to the corresponding input text(s). This allows for unprecedented interaction between text and the statistical results computed by $R$. For this paper we sampled sev- eral Wikipedia articles to present all features of $R$ integrated into TextImager. This includes articles about four politicians (Angela Merkel, Barack Obama, Recep (Tayyip) Erdoğan, Donald Trump) and five sportsman, that is, three basketball players (Michael Jordan, Kobe Bryant and Lebron James) and two soccer players (Thomas Müller and Bastian Schweinsteiger).

\section{Related Work}

$R$ is used and developed by a large community covering a wide range of statistical packages. The $C R A N^{1}$ package repository is the main repository of the $R$ project. It currently consists of about 10000 packages including packages for NLP. ${ }^{2}$ The $R$ framework requires basic to versatile skills in programming and scripting. It provides limited visualization and interaction functionalities. Attempts have been undertaken to provide web interfaces for $R$ as, for example, Shiny ${ }^{3}$ and rApache $^{4}$. Though they provide a variety of functions and visualizations ${ }^{5}$, these tools are not optimized for statistical NLP: their NLP-related functionalities are rather limited. In order to fill this gap, we introduce TextImager's $R$ package, that is, a web based tool for NLP utilizing $R$.

\section{Architecture}

TextImager is a UIMA-based (Ferrucci and Lally, 2004) framework that offers a wide range of NLP and visualization tools by means of a user-friendly GUI without requiring programming skills. It consists of two parts: front-end and back-end. The back-end is a modular, expandable, scalable and

\footnotetext{
${ }^{1}$ https: / / cran.r-project.org/

${ }^{2}$ https: / / cran.r-project.org/web/views/

Natural LanguageProcessing.html

${ }^{3}$ http: / / shiny.rstudio.com/

${ }^{4}$ http: // rapache. net /

${ }^{5}$ http://shiny.rstudio.com/gallery/
} 
flexible architecture with parallel and distributed processing capabilities (Hemati et al., 2016). The front-end is a web application that makes NLP processes available in a user-friendly way with responsive and interactive visualizations (Hemati et al., 2016). TextImager already integrated many third party tools. One of these is $R$. This section describes the technical integration and utilization of $R$ into TextImager.

\subsection{R/ OpenCPU}

$\mathrm{R}$ is a software environment for statistical computing. It compiles and runs on a variety of UNIX and Windows platforms. One of our goals is to provide an easy to use interface for R with a focus on NLP. To this end, we use $O p e n C P U$ to integrate $R$ into TextImager. OpenCPU provides an HTTP API, which allocates the functionalities of R-packages (Ooms, 2014). The OpenCPU software can be used directly in $R$; alternatively, it can be installed on a server. We decided for the latter variant. In addition, we used the opencpu.js JavaScript library which simplifies API use in JavaScript and allows for calling $R$-functions directly from TextImager. To minimize the communication effort between client and server and to encapsulate $R$ scripts from TextImager's functionality, we created a so called TextImager-R-package that takes TextImager data, performs all $R$-based calculations and returns the results. This package serves for converting any TextImager data to meet the input requirements of any $R$-package. In this way, we can easily add new packages to TextImager without changing the HTTP request code. Because some data and models have a long build time we used OpenCPU's session feature to keep this data on the server and access it in future sessions so that we do not have to recreate it. This allows the user for quickly executing several methods even in parallel without recalculating them each time.

\subsection{Data Structure}

The data structure of TextImager differs from $R$ 's data structure. Therefore, we developed a generic mapping interface that translates the data structure from TextImager to an $R$-readable format. Depending on the $R$-package, we send the required data via OpenCPU. This allows for combining each NLP tool with any $R$-package.

\subsection{OpenCPU Output Integration}

Visualizing the results of a calculation or sensitivity analysis is an important task. That is why we provide interactive visualizations to make the information available to the user more comprehensible. This allows the user to interact with the text and the output of $\mathrm{R}$, for example, by highlighting any focal sentence in a document by hovering over a word or sentence graph generated by means of $R$.

\subsection{R-packages}

This section gives an overview of $R$-packages embedded into the pipeline of TextImager.

tm The tm-package (Feinerer and Hornik, 2015) is a text mining $R$-package containing preprocess methods for data importing, corpus handling, stopword filtering and more.

Ida The lda-package (Chang, 2015) provides an implementation of Latent Dirichlet Allocation algorithms. It is used to automatically identify topics in documents, to get top documents for these topics and to predict document labels. In addition to the tabular output we developed an interactive visualization, which assigns the decisive words to the appropriate topic (see Figure 1). This visualization makes it easy to differentiate between the two topics and see which words classify these topics. In our example, we have recognized words such as players, season and game as one topic (sportsman) and party, state and politically as a different topic (politics). The parameters of every package can be set on runtime. The utilization and combination of TextImager and $R$ makes it possible, to calculate topics not only based on wordforms, but also takes other features into account like lemma, pos-tags, morphological features and more.

stylo The stylo-package (Eder et al., 2016) provides functionality for stylometric analyses. All parameters of the package can be set through the graphical user interface of TextImager. The package provides multiple unsupervised analyses, mostly based on a mostfrequent-word list and contrastive text analysis. In Figure 2 we have calculated a cluster analysis based on our example corpus. We can see that the politicians, basketball players 

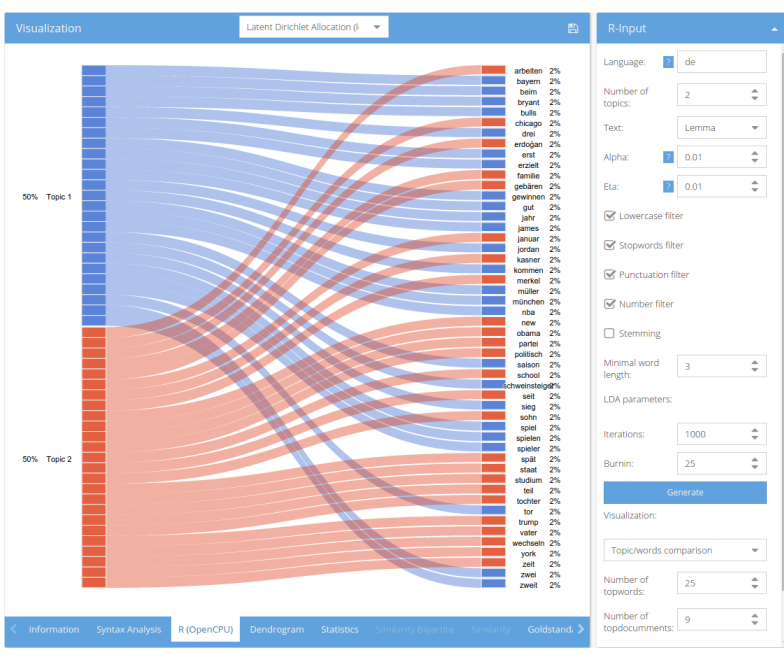

Figure 1: Words assigned to their detected topics

and soccer players have been clustered into their own groups.

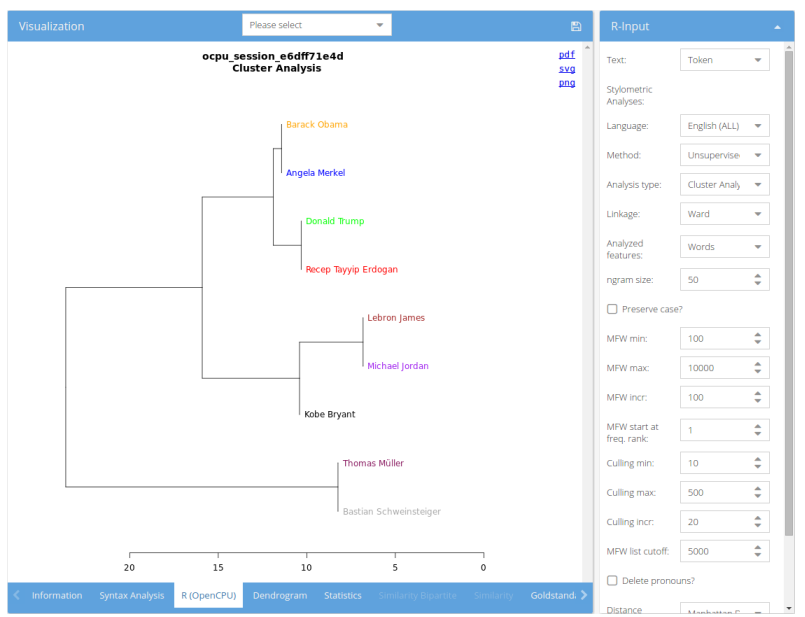

Figure 2: Cluster analysis of the documents

LSAfun The LSAfun (Günther et al., 2015) and lsa (Wild, 2015) packages provide functionality for Latent Semantic Analysis. In TextImager it is used to generate summaries of documents and similarities of sentences.

igraph The igraph-package (Csardi and Nepusz, 2006) provides multiple network analysis tools. We created a document network by linking each word with its five most similar words based on word embeddings. The igraph-package also allows to layout the graph and calculate different centrality measures. In the end, we can export the network in many formats (GraphML, GEXF, JSON, etc.) and edit it with graph editors. We also build an interactive network visualization (see figure 3) using sigma.js to make it interoperable with TextImager.

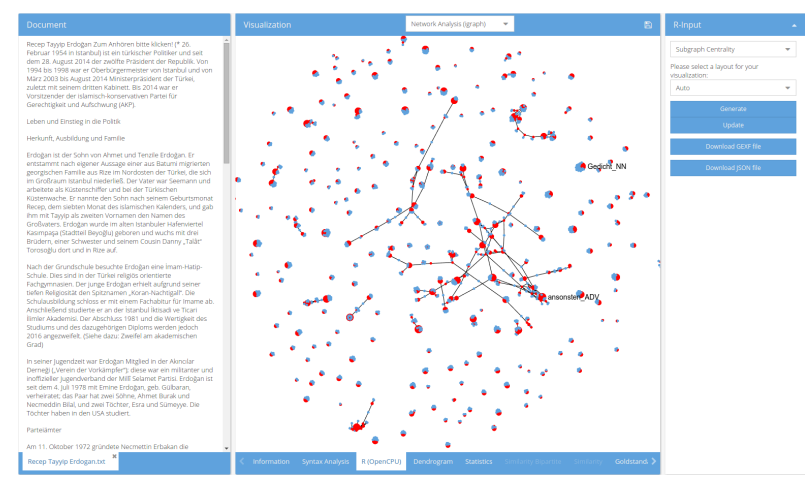

Figure 3: Network graph based on word embeddings

tidytext The tidytext package (Silge and Robinson, 2016) provides functionality to create datasets following the tidy data principles (Wickham, 2014). We used it with our $t m$ based corpus to calculate TF-IDF information of documents. In Figure 4 we see the output tabular with informations like $t f$, idf and $t f-i d f$

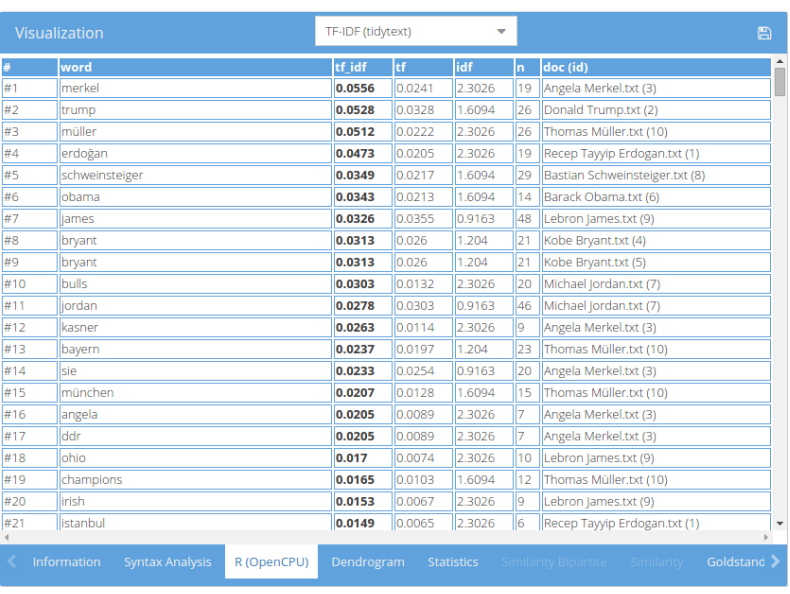

Figure 4: Statistical information of the documents

stringdist The stringdist-package (van der Loo, 2014) implements distance calculation methods, like Cosine, Jaccard, OSA and other. We implemented functionality for calculating sentence similarities and provide an interactive visual representation. Each node represents an sentence of the selected document and the links between them represent the similarity of those sentences. The thicker the links, the more similar they are. By in- 
teracting with the visualization, corresponding sections of the document panel are getting highlighted, to see the similar sentences (see Figure 5). The bidirectional interaction functionality enables easy comparability.

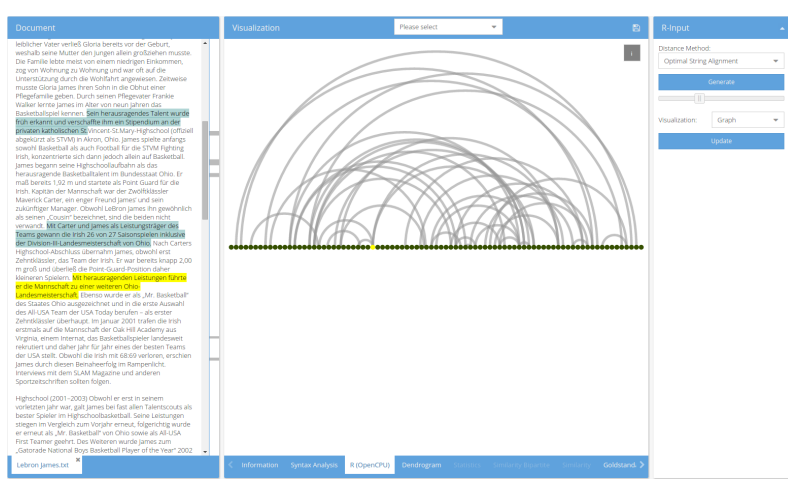

Figure 5: Depiction of sentence similarities.

stats We used functions from the $R$-package stats (R Development Core Team, 2008) to calculate a hierarchical cluster analysis based on the sentence similarities. This allows us to cluster similar sentences and visualize them with an interactive dendrogram. In Figure 6 we selected on one of these clusters and the document panel immediately adapts and highlights all the sentences in this cluster.

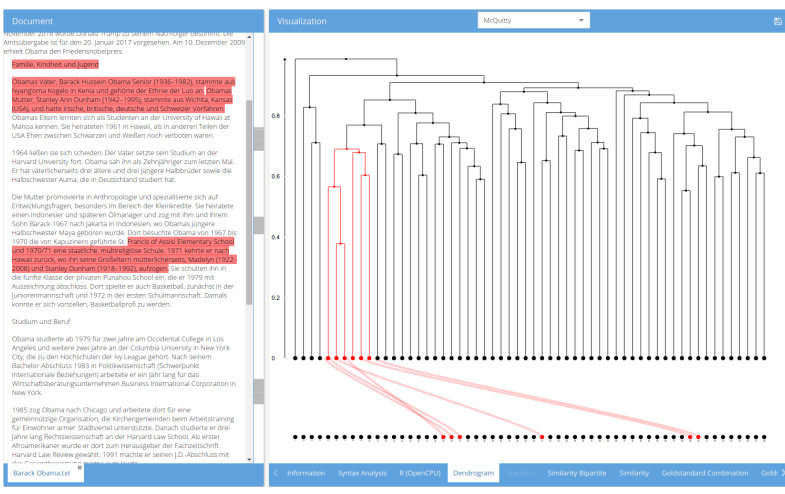

Figure 6: Similarity-clustered sentences.

An interesting side effect of integrating these tools into TextImager's pipeline is that their output can be concerted in a way to arrive at higherlevel text annotations and analyses. In this way, we provide to an integration of two heavily expanding areas, that is, NLP and statistical modeling.

\section{Future work}

In already ongoing work, we focus on big data as exemplified by the Wikipedia. We also extend the number of built-in $R$-packages in TextImager.

\section{Scope of the Software Demonstration}

The beta version of TextImager is online. To test the functionalities of $R$ as integrated into TextImager use the following demo: http: //textimager.hucompute.org/ index.html?viewport=demo\&file= $\mathrm{R}$-Demo.xml.

\section{References}

Jonathan Chang, 2015. lda: Collapsed Gibbs Sampling Methods for Topic Models. R package version 1.4.2.

Gabor Csardi and Tamas Nepusz. 2006. The igraph software package for complex network research. InterJournal, Complex Systems:1695.

Maciej Eder, Jan Rybicki, and Mike Kestemont. 2016. Stylometry with R: a package for computational text analysis. R Journal, 8(1):107-121.

Ingo Feinerer and Kurt Hornik, 2015. tm: Text Mining Package. R package version 0.6-2.

David Ferrucci and Adam Lally. 2004. UIMA: an architectural approach to unstructured information processing in the corporate research environment. Natural Language Engineering, 10(3-4):327-348.

Fritz Günther, Carolin Dudschig, and Barbara Kaup. 2015. Lsafun: An R package for computations based on latent semantic analysis. Behavior Research Methods, 47(4):930-944.

Wahed Hemati, Tolga Uslu, and Alexander Mehler. 2016. Textimager: a distributed UIMA-based system for NLP. In Proceedings of the COLING 2016 System Demonstrations.

Jeroen Ooms. 2014. The OpenCPU system: Towards a universal interface for scientific computing through separation of concerns. arXiv:1406.4806 [stat.CO].

R Development Core Team, 2008. R: A Language and Environment for Statistical Computing. R Foundation for Statistical Computing, Vienna, Austria. ISBN 3-900051-07-0.

Julia Silge and David Robinson. 2016. tidytext: Text mining and analysis using tidy data principles in $\mathrm{R}$. JOSS, 1(3).

M.P.J. van der Loo. 2014. The stringdist package for approximate string matching. The R Journal, 6:111122.

Hadley Wickham. 2014. Tidy data. Journal of Statistical Software, 59(1):1-23.

Fridolin Wild, 2015. lsa: Latent Semantic Analysis. R package version 0.73 .1 . 